\title{
A micropalaeontological fraud that affected the JAES
}

\author{
Bruno Granier $^{\text {a, }}$, Monique Feist ${ }^{b}$, Patrick G. Eriksson ${ }^{c}$ and Sospeter M. Muhongo ${ }^{d}$
}

a Département des Sciences de la Terre et de l'Univers, UFR des Sciences et Techniques, Université de Bretagne Occidentale (UBO), 6 avenue Le Gorgeu, F29238 Brest Cedex 3, France

b Laboratoire de Paléontologie (C.C.62), Université Montpellier 2, Place Eugène Bataillon, F-34095 Montpellier Cedex 05, France

c Department of Geology, University of Pretoria, Pretoria 0002, South Africa

d Regional Office for Africa International Council for Science (ICSU), PO Box 13252, Hatfield, Pretoria, South Africa

\section{Article Outline}

1. Introduction

2.Phillip et al. (1997)

3. Refaat and Imam (1999)

4. Imam (1999)

5. Imam (2001)

6. Conclusions

Acknowledgements

References

\section{Introduction}

Although one is loath to publish facts that tarnish the reputation of a colleague in science, and understanding that inexperienced researchers may inadvertently or through ignorance be guilty of a minor plagiarism, occasional cases of what might be termed "serial plagiarisers" become apparent. In such cases, there is little choice but to expose the person, not to do them a personal injury, but rather to ensure that the damage done to science becomes known and thus that potential citation of a false paper can be avoided, or at least minimised. It is a remarkable facet of African and Middle-Eastern geosciences, that despite widespread poverty and a common lack of resources to pursue research, that good scientific work is in fact done almost everywhere, in the face of such negative factors. The tarnishing of African-Middle Eastern geological endeavour by a very small number of individuals cannot be allowed to dilute the dedication and contribution made by the over-riding majority of geoscience workers in this vast region served by the Journal of African Earth Sciences. It is thus with profound regret and sadness that the 
following report must needs be given; there is no satisfaction to be had in such a necessity, nor is vindictiveness intended.

After the 8th International Symposium on Fossil Algae held in Granada, Spain (September 18-20, 2003), a set of conference papers as well as some additional manuscripts were considered for publication in a dedicated volume of the Spanish journal, Revista Española de Micropaleontologia. Aguirre (2004), one of the two special editors, was given one manuscript to peer-review. At first sight he found that the material illustrated by the author, Mostafa Mansour Imam, was very familiar to him. Then he realized that some photomicrographs, mostly those illustrating fossil coralline algae, were duplicates of figures from his own publications, from those of his colleagues, and from the classical series of papers published from the late 1950s to the early 1970s by J.H. Johnson. Finally, he found that the fraud also included illustrations from three earlier papers published in the Revista Española de Micropaleontologia and in the German journal, Neues Jahrbuch für Geologie und Paläontologie, Abhandlungen. Once aware of the full extent of the fraud, Aguirre decided to warn the scientific community and published a short report (Aguirre, 2004) revealing the falsification; he also announced that a group (the authors of this report) were still investigating the case.

During the period 1996-2003, M.M. Imam published four papers in the Journal of African Earth Sciences: two as junior author ([Phillip et al., 1997] and [Refaat and Imam, 1999]), then two as the sole author ([Imam, 1999] and [Imam, 2001]). The details of plagiarised material in these papers are given below.

\section{Phillip et al. (1997)}

M.M. Imam was the second author of this multi-authored paper. Some photomicrographs from Youssef et al. (1988) were re-used, but are valid reproductions, for both papers investigate the same locality. However, Figs. 5-7 of his Pl. 1 (that is Figs. 4.5-4.7) of Phillip et al. (1997) are mirror-image views of the original photomicrographs in Youssef et al. (1988): a left-coiling specimen is converted into a right-coiling one, and vice-versa.

\begin{tabular}{|l|l|l|l|}
\hline \multicolumn{2}{|l|}{ Phillip et al. (1997) } & \multicolumn{2}{l|}{ Youssef et al. (1988) } \\
\hline $\begin{array}{l}\text { Pl. 1, Fig. 7 (Fig. } \\
\text { 4.7) }\end{array}$ & Globigerina ciperoensis ciperoensis & $\begin{array}{l}\text { Fig. } \\
5 \mathrm{~A}\end{array}$ & Globigerina ciperoensis \\
\hline $\begin{array}{l}\text { Pl. 2, Fig. 3 (Fig. } \\
\text { 5.3) }\end{array}$ & Globigerinoides primordius & $\begin{array}{l}\text { Fig. } \\
5 \mathrm{~B}\end{array}$ & $\begin{array}{l}\text { Globigerinoides cf. } \\
\text { primordius }\end{array}$ \\
\hline $\begin{array}{l}\text { Pl. 1, Fig. 6 (Fig. } \\
\text { 4.6) }\end{array}$ & $\begin{array}{l}\text { Globigerina ciperoensis } \\
\text { angustiumbilicata }\end{array}$ & $\begin{array}{l}\text { Fig. } \\
5 \mathrm{C}\end{array}$ & $\begin{array}{l}\text { Globigerina } \\
\text { angustiumbilicata }\end{array}$ \\
\hline $\begin{array}{l}\text { Pl. 1, Fig. 5 (Fig. } \\
4.5)\end{array}$ & Globigerina eamesi & $\begin{array}{l}\text { Fig. } \\
5 \mathrm{D}\end{array}$ & Globigerina bulloides \\
\hline
\end{tabular}




\section{Refaat and Imam (1999)}

This paper deals with charophyte remains supposedly collected in Upper Eocene strata in Sinai, Egypt. Figs. 9 and 10 comprise, respectively, 22 and 16 gyrogonites. All of these 38 images were "borrowed" from 4 publications ([Feist-Castel, 1977], [Feist and Ringeade, 1977], [Grambast and Grambast-Fessard, 1981] and [Grambast-Fessard, 1980]). The results of our investigation are summarized in the four following tables:

\begin{tabular}{|c|c|c|c|c|}
\hline \multicolumn{2}{|c|}{ Refaat and Imam (1999) } & \multicolumn{3}{|c|}{ Grambast-Fessard (1980) } \\
\hline Fig. 9.1 & Harrisichara lineate & Pl. IV, Fig. 7 & Harrisichara heteromorpha $\mathrm{n} . \mathrm{sp}$. & Palaeocene \\
\hline Fig. 9.2 & Harrisichara lineate & Pl. IV, Fig. 9 & $\begin{array}{l}\text { Harrisichara heteromorpha n.sp. } \\
\text { (Holotype) }\end{array}$ & Palaeocene \\
\hline Fig. 9.3 & Harrisichara lineate & Pl. IV, Fig. 8 & Harrisichara heteromorpha n.sp. & Palaeocene \\
\hline Fig. 9.4 & Harrisichara tuberculata & Pl. IV, Fig. 2 & $\begin{array}{l}\text { Harrisichara muricata n.sp. } \\
\text { (Holotype) }\end{array}$ & Palaeocene \\
\hline Fig. 9.5 & Harrisichara tuberculata & Pl. IV, Fig. 1 & Harrisichara muricata n.sp. & Palaeocene \\
\hline Fig. 9.6 & Harrisichara cf. tuberculata & Pl. III, Fig. 2 & $\begin{array}{l}\text { Harrisichara regularis n.sp. } \\
\text { (Holotype) }\end{array}$ & Palaeocene \\
\hline Fig. 9.7 & Harrisichara cf. tuberculata & Pl. III, Fig. 3 & Harrisichara regularis n.sp. & Palaeocene \\
\hline Fig. 9.8 & $\begin{array}{l}\text { Harrisichara vasiformis- } \\
\text { tuberculata }\end{array}$ & Pl. III, Fig. 1 & Harrisichara regularis n.sp. & Palaeocene \\
\hline Fig. 9.9 & $\begin{array}{l}\text { Harrisichara vasiformis- } \\
\text { tuberculata }\end{array}$ & Pl. IV, Fig. 3 & Harrisichara muricata n.sp. & Palaeocene \\
\hline Fig. 9.10 & $\begin{array}{l}\text { Harrisichara vasiformis- } \\
\text { tuberculata }\end{array}$ & Pl. IV, Fig. 6 & $\begin{array}{l}\text { Harrisichara muricata n.sp. } \\
\text { (Holotype) }\end{array}$ & Palaeocene \\
\hline Fig. 9.11 & Nitellopsis meriani & Pl. II, Fig. 1 & $\begin{array}{l}\text { Nitellopsis helicteres minor } \\
\text { n.ssp. (Holotype) }\end{array}$ & Palaeocene \\
\hline Fig. 9.12 & Nitellopsis meriani & Pl. II, Fig. 4 & $\begin{array}{l}\text { Nitellopsis helicteres minor } \\
\text { n.ssp. (Holotype) }\end{array}$ & Palaeocene \\
\hline Fig. 9.13 & Nitellopsis meriani & Pl. II, Fig. 2 & $\begin{array}{l}\text { Nitellopsis helicteres minor } \\
\text { n.ssp. }\end{array}$ & Palaeocene \\
\hline Fig. 9.14 & Nitellopsis meriani & Pl. II, Fig. 3 & $\begin{array}{l}\text { Nitellopsis helicteres minor } \\
\text { n.ssp. (Holotype) }\end{array}$ & Palaeocene \\
\hline $\begin{array}{l}\text { Fig. } \\
10.16\end{array}$ & Rhabdochara stockmansis & Pl. I, Fig. 6 & Dughiella ovoidea & Palaeocene \\
\hline \multicolumn{2}{|c|}{ Refaat and Imam (1999) } & \multicolumn{3}{|c|}{ Grambast and Grambast-Fessard (1981) } \\
\hline Fig. 9.15 & Rhabdochara cf. altilis & Pl. III, Fig. 1 & $\begin{array}{l}\text { Gyrogona morelleti n.sp. } \\
\text { (Holotype) }\end{array}$ & $\begin{array}{l}\text { Middle-Upper } \\
\text { Eocene }\end{array}$ \\
\hline
\end{tabular}




\begin{tabular}{|c|c|c|c|c|}
\hline \multicolumn{2}{|c|}{ Refaat and Imam (1999) } & \multicolumn{3}{|c|}{ Grambast-Fessard (1980) } \\
\hline Fig. 9.16 & Rhabdochara cf. altilis & Pl. I, Fig. 3 & Gyrogona lamarcki n.sp. & $\begin{array}{l}\text { Middle-Upper } \\
\text { Eocene }\end{array}$ \\
\hline Fig. 9.17 & Gyrogona caelata & Pl. I, Fig. 13 & $\begin{array}{l}\text { Gyrogona lemani capitata n.ssp. } \\
\text { (Holotype) }\end{array}$ & $\begin{array}{l}\text { Middle-Upper } \\
\text { Eocene }\end{array}$ \\
\hline $\begin{array}{l}\text { Fig. } \\
10.13\end{array}$ & Gyrogona subglobosa & Pl. II, Fig. 2 & Gyrogona lamarcki n.sp. & $\begin{array}{l}\text { Middle-Upper } \\
\text { Eocene }\end{array}$ \\
\hline $\begin{array}{l}\text { Fig. } \\
10.14\end{array}$ & Gyrogona subglobosa & Pl. I, Fig. 1 & $\begin{array}{l}\text { Gyrogona lamarcki n.sp. } \\
\text { (Holotype) }\end{array}$ & $\begin{array}{l}\text { Middle-Upper } \\
\text { Eocene }\end{array}$ \\
\hline $\begin{array}{l}\text { Fig. } \\
10.15\end{array}$ & Gyrogona subglobosa & Pl. I, Fig. 7 & Gyrogona lamarcki n.sp. & $\begin{array}{l}\text { Middle-Upper } \\
\text { Eocene }\end{array}$ \\
\hline \multicolumn{2}{|c|}{ Refaat and Imam (1999) } & \multicolumn{3}{|c|}{ Feist and Ringeade (1977) } \\
\hline Fig. 9.18 & Sphaerochara subglobosa & Pl. XII, Fig. 9 & Sphaerocharaaff. davidi & Lower Miocene \\
\hline Fig. 9.19 & Sphaerochara ulmensis & P1. XIII, Fig. 7 & Sphaerocharaaff. davidi & Lower Miocene \\
\hline Fig. 9.20 & Stephanochara ungeri & Pl. XIII, Fig. 2 & Stephanochara berdotensis n.sp. & Lower Miocene \\
\hline Fig. 9.21 & Stephanochara ungeri & Pl. XIII, Fig. 3 & Stephanochara berdotensis n.sp. & Lower Miocene \\
\hline Fig. 9.22 & Stephanochara ungeri & Pl. XIII, Fig. 6 & Stephanochara berdotensis n.sp. & Lower Miocene \\
\hline Fig. 10.7 & Rhabdochara major & Pl. XIII, Fig. 9 & Rhabdochara langeri & $\begin{array}{l}\text { Oligocene-- } \\
\text { Lower Miocene }\end{array}$ \\
\hline Fig. 10.8 & Chara notata & Pl. XII, Fig. 7 & Chara notata & $\begin{array}{l}\text { Oligocene-- } \\
\text { Lower Miocene }\end{array}$ \\
\hline Fig. 10.9 & $\begin{array}{l}\text { Harrisichara vasiformis- } \\
\text { tuberculata }\end{array}$ & Pl. XI, Fig. 3 & Sphaerochara labellala n.sp. & Upper Eocene \\
\hline $\begin{array}{l}\text { Fig. } \\
10.10\end{array}$ & $\begin{array}{l}\text { Harrisichara vasiformis- } \\
\text { tuberculata }\end{array}$ & Pl. XI, Fig. 2 & Sphaerochara labellala n.sp. & Upper Eocene \\
\hline $\begin{array}{l}\text { Fig. } \\
10.11\end{array}$ & Harrisichara sp. & Pl. X, Fig. 10 & Harrisichara subteres n.sp. & Upper Eocene \\
\hline $\begin{array}{l}\text { Fig. } \\
10.12\end{array}$ & Harrisichara sp. & P1. X, Fig. 12 & Harrisichara subteres n.sp. & Upper Eocene \\
\hline \multicolumn{2}{|c|}{ Refaat and Imam (1999) } & \multicolumn{3}{|c|}{ Feist-Castel (1977) } \\
\hline Fig. 10.1 & Nitellopsis meriani & Pl. I, Fig. 3 & $\begin{array}{l}\text { Nitellopsis (Microstomella) } \\
\text { aptensis n.sp. (Holotype) }\end{array}$ & $\begin{array}{l}\text { Upper Eocene- } \\
\text { Lower } \\
\text { Oligocene }\end{array}$ \\
\hline Fig. 10.2 & Harrisichara tuberculata & Pl. IV, Fig. 1 & $\begin{array}{l}\text { Sphaerochara davidi n.sp. } \\
\text { (Holotype) }\end{array}$ & $\begin{array}{l}\text { Upper } \\
\text { Oligocene }\end{array}$ \\
\hline Fig. 10.3 & Harrisichara tuberculata & Pl. IV, Fig. 4 & Sphaerochara davidi n.sp. & Upper \\
\hline
\end{tabular}




\begin{tabular}{|l|l|l|l|l|}
\hline \multicolumn{2}{|l|}{ Refaat and Imam (1999) } & Grambast-Fessard (1980) & Oligocene \\
\hline & & & & $\begin{array}{l}\text { Upper } \\
\text { Oligocene }\end{array}$ \\
\hline Fig. 10.4 & Stephanochara berdotensis & Pl. IV, Fig. 7 & $\begin{array}{l}\text { Stephanochara oodea } \text { n.sp. } \\
\text { (Holotype) }\end{array}$ & $\begin{array}{l}\text { Upper } \\
\text { Oligocene }\end{array}$ \\
\hline Fig. 10.5 & Stephanochara berdotensis & Pl. IV, Fig. 10 & Stephanochara oodea n.sp. & $\begin{array}{l}\text { Upper } \\
\text { Oligocene }\end{array}$ \\
\hline Fig. 10.6 & Stephanochara vectensis & Pl. IV, Fig. 9 & Stephanochara oodea n.sp. & \\
\hline
\end{tabular}

With the exception of Fig. 10.8, all denominations of already published figures have been changed. Most images illustrated types (paratypes and even holotypes) and in a few cases (Figs. 9.15 and 10.6-7) gyrogonites were rotated $180^{\circ}$ and consequently appear with their bases upward, thus demonstrating that the author is not aware of the standards or conventions used by specialists of this particular microfossil group.

\section{Imam (1999)}

This paper deals with planktonic foraminifera supposedly collected in strata of Late Eocene to Middle Miocene age from northeastern Libya. We did not find the "source" of the 16 photomicrographs used in Fig. 7, but we identify all the images (32) used in Figs. 8 and 9 which were "borrowed" from one publication (Waters and Snyder, 1986). The results of our investigation are summarized in the following table:

\begin{tabular}{|c|c|c|c|c|c|}
\hline \multicolumn{3}{|c|}{ Imam (1999) } & \multicolumn{3}{|c|}{ Waters and Snyder (1986) } \\
\hline Fig. 8.1 & $\begin{array}{l}\text { Cassigerinella } \\
\text { chiploensis }\end{array}$ & Lower Miocene & Pl. 4, Fig. H & $\begin{array}{l}\text { Cassigerinella } \\
\text { chipolensis }\end{array}$ & $\begin{array}{l}\text { Upper } \\
\text { Oligocene- } \\
\text { Middle } \\
\text { Miocene }\end{array}$ \\
\hline Fig. 8.2 & $\begin{array}{l}\text { Catapsydrax } \\
\text { dissimilis }\end{array}$ & $\begin{array}{l}\text { Upper Oligocene- } \\
\text { Lower Miocene }\end{array}$ & Pl. 4, Fig. N & $\begin{array}{l}\text { Catapsydrax } \\
\text { dissimilis }\end{array}$ & $\begin{array}{l}\text { Upper } \\
\text { Oligocene- } \\
\text { Lower } \\
\text { Miocene }\end{array}$ \\
\hline Fig. 8.3 & $\begin{array}{l}\text { Globigerina } \\
\text { ciperoensis } \\
\text { ciperoensis }\end{array}$ & $\begin{array}{l}\text { Upper Oligocene- } \\
\text { Lower Miocene }\end{array}$ & Pl. 3, Fig. E & $\begin{array}{l}\text { Globigerina } \\
\text { ciperoensis }\end{array}$ & $\begin{array}{l}\text { Upper } \\
\text { Oligocene }\end{array}$ \\
\hline Fig. 8.4 & $\begin{array}{l}\text { Globigerina } \\
\text { ciperoensis } \\
\text { ciperoensis }\end{array}$ & $\begin{array}{l}\text { Upper Oligocene- } \\
\text { Lower Miocene }\end{array}$ & Pl. 3, Fig. G & $\begin{array}{l}\text { Globigerina } \\
\text { ciperoensis }\end{array}$ & $\begin{array}{l}\text { Upper } \\
\text { Oligocene }\end{array}$ \\
\hline Fig. 8.5 & $\begin{array}{l}\text { Globigerina } \\
\text { ciperoensis } \\
\text { angustiumbilicat } \\
a\end{array}$ & Upper Oligocene & Pl. 3, Fig. R & $\begin{array}{l}\text { Globigerina } \\
\text { angustiumbilicata }\end{array}$ & $\begin{array}{l}\text { Upper } \\
\text { Oligocene- } \\
\text { Middle } \\
\text { Miocene }\end{array}$ \\
\hline
\end{tabular}




\begin{tabular}{|c|c|c|c|c|c|}
\hline \multicolumn{3}{|c|}{ Imam (1999) } & \multicolumn{3}{|c|}{ Waters and Snyder (1986) } \\
\hline Fig. 8.6 & $\begin{array}{l}\text { Globigerina } \\
\text { angulisuturalis }\end{array}$ & $\begin{array}{l}\text { Upper Oligocene- } \\
\text { Lower Miocene }\end{array}$ & Pl. 3, Fig. J & $\begin{array}{l}\text { Globigerina } \\
\text { pseudociperoensis }\end{array}$ & $\begin{array}{l}\text { Lower-Middle } \\
\text { Miocene }\end{array}$ \\
\hline Fig. 8.7 & $\begin{array}{l}\text { Globorotalia } \\
\text { siakensis }\end{array}$ & Lower Miocene & Pl. 2, Fig. T & $\begin{array}{l}\text { Globorotalia } \\
\text { siakensis }\end{array}$ & $\begin{array}{l}\text { Upper } \\
\text { Oligocene- } \\
\text { Middle } \\
\text { Miocene }\end{array}$ \\
\hline Fig. 8.8 & $\begin{array}{l}\text { Globorotalia } \\
\text { siakensis }\end{array}$ & Lower Miocene & Pl. 2, Fig. S & $\begin{array}{l}\text { Globorotalia } \\
\text { siakensis }\end{array}$ & $\begin{array}{l}\text { Upper } \\
\text { Oligocene- } \\
\text { Middle } \\
\text { Miocene }\end{array}$ \\
\hline Fig. 8.9 & $\begin{array}{l}\text { Globigerina } \\
\text { praebulloides }\end{array}$ & $\begin{array}{l}\text { Upper Oligocene- } \\
\text { Lower Miocene }\end{array}$ & Pl. 3, Fig. P & $\begin{array}{l}\text { Globorotalia scitula } \\
\text { praescitula }\end{array}$ & $\begin{array}{l}\text { Lower-Middle } \\
\text { Miocene }\end{array}$ \\
\hline Fig. 8.10 & $\begin{array}{l}\text { Globigerina } \\
\text { praebulloides }\end{array}$ & $\begin{array}{l}\text { Upper Oligocene- } \\
\text { Lower Miocene }\end{array}$ & Pl. 3, Fig. Q & $\begin{array}{l}\text { Globorotalia scitula } \\
\text { praescitula }\end{array}$ & $\begin{array}{l}\text { Lower-Middle } \\
\text { Miocene }\end{array}$ \\
\hline Fig. 8.11 & $\begin{array}{l}\text { Globorotalia } \\
\text { kugleri }\end{array}$ & $\begin{array}{l}\text { Oligocene-Miocene } \\
\text { transition }\end{array}$ & Pl. 2, Fig. K & Globorotalia kugleri & $\begin{array}{l}\text { Oligocene- } \\
\text { Miocene } \\
\text { transition }\end{array}$ \\
\hline Fig. 8.12 & $\begin{array}{l}\text { Globorotalia } \\
\text { kugleri }\end{array}$ & $\begin{array}{l}\text { Oligocene-Miocene } \\
\text { transition }\end{array}$ & Pl. 2, Fig. J & Globorotalia kugleri & $\begin{array}{l}\text { Oligocene- } \\
\text { Miocene } \\
\text { transition }\end{array}$ \\
\hline Fig. 8.13 & $\begin{array}{l}\text { Globorotalia } \\
\text { obesa }\end{array}$ & Lower Miocene & Pl. 2, Fig. L & Globorotalia obesa & $\begin{array}{l}\text { Upper } \\
\text { Oligocene- } \\
\text { Middle } \\
\text { Miocene }\end{array}$ \\
\hline Fig. 8.14 & $\begin{array}{l}\text { Globorotalia } \\
\text { obesa }\end{array}$ & Lower Miocene & Pl. 2, Fig. N & Globorotalia obesa & $\begin{array}{l}\text { Upper } \\
\text { Oligocene- } \\
\text { Middle } \\
\text { Miocene }\end{array}$ \\
\hline Fig. 8.15 & $\begin{array}{l}\text { Globigerina } \\
\text { venezuelana }\end{array}$ & $\begin{array}{l}\text { Upper Oligocene-- } \\
\text { Lower Miocene }\end{array}$ & Pl. 3, Fig. M & $\begin{array}{l}\text { Globigerina } \\
\text { venezuelana }\end{array}$ & $\begin{array}{l}\text { Upper } \\
\text { Oligocene- } \\
\text { Middle } \\
\text { Miocene }\end{array}$ \\
\hline Fig. 8.16 & $\begin{array}{l}\text { Globigerina } \\
\text { venezuelana }\end{array}$ & $\begin{array}{l}\text { Upper Oligocene- } \\
\text { Lower Miocene }\end{array}$ & Pl. 3, Fig. N & $\begin{array}{l}\text { Globigerina } \\
\text { venezuelana }\end{array}$ & $\begin{array}{l}\text { Upper } \\
\text { Oligocene- } \\
\text { Middle } \\
\text { Miocene }\end{array}$ \\
\hline Fig. 9.1 & $\begin{array}{l}\text { Globigerinoides } \\
\text { primordius }\end{array}$ & Lower Miocene & Pl. 1, Fig. J & $\begin{array}{l}\text { Globigerinoides } \\
\text { quadrilobatus } \\
\text { primordius }\end{array}$ & $\begin{array}{l}\text { Lower } \\
\text { Miocene }\end{array}$ \\
\hline Fig. 9.2 & $\begin{array}{l}\text { Globigerinoides } \\
\text { primordius }\end{array}$ & Lower Miocene & Pl. 1, Fig. K & $\begin{array}{l}\text { Globigerinoides } \\
\text { quadrilobatus } \\
\text { primordius }\end{array}$ & $\begin{array}{l}\text { Lower } \\
\text { Miocene }\end{array}$ \\
\hline
\end{tabular}




\begin{tabular}{|c|c|c|c|c|c|}
\hline \multicolumn{3}{|c|}{ Imam (1999) } & \multicolumn{3}{|c|}{ Waters and Snyder (1986) } \\
\hline Fig. 9.3 & $\begin{array}{l}\text { Globigerinoides } \\
\text { trilobus }\end{array}$ & Lower Miocene & Pl. 1, Fig. F & $\begin{array}{l}\text { Globigerinoides } \\
\text { quadrilobatus triloba }\end{array}$ & $\begin{array}{l}\text { Lower-Middle } \\
\text { Miocene }\end{array}$ \\
\hline Fig. 9.4 & $\begin{array}{l}\text { Globigerinoides } \\
\text { trilobus }\end{array}$ & Lower Miocene & Pl. 1, Fig. G & $\begin{array}{l}\text { Globigerinoides } \\
\text { quadrilobatus triloba }\end{array}$ & $\begin{array}{l}\text { Lower-Middle } \\
\text { Miocene }\end{array}$ \\
\hline Fig. 9.5 & $\begin{array}{l}\text { Globigerinoides } \\
\text { immaturus }\end{array}$ & Lower Miocene & Pl. 1, Fig. H & $\begin{array}{l}\text { Globigerinoides } \\
\text { quadrilobatus } \\
\text { praeimmaturus }\end{array}$ & $\begin{array}{l}\text { Lower-Middle } \\
\text { Miocene }\end{array}$ \\
\hline Fig. 9.6 & $\begin{array}{l}\text { Globigerinoides } \\
\text { immaturus }\end{array}$ & Lower Miocene & Pl. 1, Fig. I & $\begin{array}{l}\text { Globigerinoides } \\
\text { quadrilobatus } \\
\text { praeimmaturus }\end{array}$ & $\begin{array}{l}\text { Lower-Middle } \\
\text { Miocene }\end{array}$ \\
\hline Fig. 9.7 & $\begin{array}{l}\text { Globigerinoides } \\
\text { sacculifer }\end{array}$ & Lower Miocene & Pl. 1, Fig. D & $\begin{array}{l}\text { Globigerinoides } \\
\text { quadrilobatus } \\
\text { sacculifer }\end{array}$ & $\begin{array}{l}\text { Lower-Middle } \\
\text { Miocene }\end{array}$ \\
\hline Fig. 9.8 & $\begin{array}{l}\text { Globigerinoides } \\
\text { sacculifer }\end{array}$ & Lower Miocene & Pl. 1, Fig. E & $\begin{array}{l}\text { Globigerinoides } \\
\text { quadrilobatus } \\
\text { sacculifer }\end{array}$ & $\begin{array}{l}\text { Lower-Middle } \\
\text { Miocene }\end{array}$ \\
\hline Fig. 9.9 & $\begin{array}{l}\text { Globigerinoides } \\
\text { subquadratus }\end{array}$ & Lower Miocene & Pl. 1, Fig. N & $\begin{array}{l}\text { Globigerinoides } \\
\text { subquadratus }\end{array}$ & $\begin{array}{l}\text { Lower-Middle } \\
\text { Miocene }\end{array}$ \\
\hline Fig. 9.10 & $\begin{array}{l}\text { Globigerinoides } \\
\text { altiaperturus }\end{array}$ & Lower Miocene & Pl. 1, Fig. A & $\begin{array}{l}\text { Globigerinoides } \\
\text { quadrilobatus } \\
\text { altiapertura }\end{array}$ & $\begin{array}{l}\text { Lower } \\
\text { Miocene }\end{array}$ \\
\hline Fig. 9.11 & $\begin{array}{l}\text { Globigerinoides } \\
\text { altiaperturus }\end{array}$ & Lower Miocene & Pl. 1, Fig. B & $\begin{array}{l}\text { Globigerinoides } \\
\text { quadrilobatus } \\
\text { altiapertura }\end{array}$ & $\begin{array}{l}\text { Lower } \\
\text { Miocene }\end{array}$ \\
\hline Fig. 9.12 & $\begin{array}{l}\text { Globigerinoides } \\
\text { sicanus }\end{array}$ & Lower Miocene & Pl. 1, Fig. L & $\begin{array}{l}\text { Globigerinoides } \\
\text { sicanus praesicanus }\end{array}$ & $\begin{array}{l}\text { Lower-Middle } \\
\text { Miocene }\end{array}$ \\
\hline Fig. 9.13 & $\begin{array}{l}\text { Globigerinoides } \\
\text { woodi }\end{array}$ & & Pl. 3, Fig. H & $\begin{array}{l}\text { Globigerinoides } \\
\text { woodi }\end{array}$ & $\begin{array}{l}\text { Upper } \\
\text { Oligocene }\end{array}$ \\
\hline Fig. 9.14 & $\begin{array}{l}\text { Globoquadrina } \\
\text { dehiscens } \\
\text { dehiscens }\end{array}$ & Lower Miocene & Pl. 4, Fig. A & $\begin{array}{l}\text { Globoquadrina } \\
\text { dehiscens }\end{array}$ & $\begin{array}{l}\text { Lower-Middle } \\
\text { Miocene }\end{array}$ \\
\hline Fig. 9.15 & $\begin{array}{l}\text { Globigerinoides } \\
\text { altispira globosa }\end{array}$ & Lower Miocene & Pl. 3, Fig. O & $\begin{array}{l}\text { Globigerina } \\
\text { venezuelana }\end{array}$ & $\begin{array}{l}\text { Upper } \\
\text { Oligocene- } \\
\text { Middle } \\
\text { Miocene }\end{array}$ \\
\hline Fig. 9.16 & $\begin{array}{l}\text { Globigerinoides } \\
\text { altispira altispira }\end{array}$ & Lower Miocene & Pl. 4, Fig. G & $\begin{array}{l}\text { Globoquadrina } \\
\text { altispira globosa }\end{array}$ & $\begin{array}{l}\text { Upper } \\
\text { Oligocene-- } \\
\text { Middle } \\
\text { Miocene }\end{array}$ \\
\hline
\end{tabular}




\section{Imam (2001)}

This paper deals with planktonic foraminifera supposedly collected in Upper Cretaceous to Lower Eocene strata from northeastern Libya. Except for Fig. 6.6 ("Abathomphalus mayaroensis"), we identify all remaining images (25) used in Fig. 6 which were "borrowed" from one publication (Petters, 1983).

\begin{tabular}{|c|c|c|c|}
\hline \multicolumn{2}{|c|}{ Imam (2001) } & \multicolumn{2}{|l|}{ Petters (1983) } \\
\hline Fig. 6.1 & Heterohelix striata & Pl. 1, Fig. 14 & Heterohelix striata \\
\hline Fig. 6.2 & Heterohelix striata & Pl. 1, Fig. 15 & Heterohelix striata \\
\hline Fig. 6.3 & Pseudogumbelina excolata [sic] & Pl. 1, Fig. 16 & Pseudoguembelina costulata \\
\hline Fig. 6.4 & Heterohelix navarroensis & Pl. 1, Fig. 21 & Heterohelix navarroensis \\
\hline Fig. 6.5 & Pseudotextularia eleganus [sic] & Pl. 1, Fig. 13 & Pseudotextularia elegans \\
\hline Fig. 6.7 & Globotruncana aegyptiaca & Pl. 2, Fig. 3 & Globotruncana aegyptiaca \\
\hline Fig. 6.8 & Globotruncana aegyptiaca & Pl. 2, Fig. 4 & Globotruncana aegyptiaca \\
\hline Fig. 6.9 & Gansserina gansseri & Pl. 2, Fig. 1 & Globotruncana gansseri \\
\hline Fig. 6.10 & Gansserina gansseri & Pl. 2, Fig. 2 & Globotruncana gansseri \\
\hline Fig. 6.11 & Rugoglobigerina rugosa & Pl. 2, Fig. 5 & Rugoglobigerina macrocephala \\
\hline Fig. 6.12 & Rugoglobigerina rugosa & Pl. 2, Fig. 9 & Rugoglobigerina macrocephala \\
\hline Fig. 6.13 & Globigerinelloides asperus & Pl. 2, Fig. 19 & Globigerinelloides caseyi \\
\hline Fig. 6.14 & Morozovella angulata & Pl. 5, Fig. 19 & Morozovella angulata \\
\hline Fig. 6.15 & Praemurica uncinata & Pl. 5, Fig. 6 & Morozovella gracilis \\
\hline Fig. 6.16 & Globanomalina compressa & Pl. 3, Fig. 12 & Planorotalites compressa \\
\hline Fig. 6.17 & Igorina pusilla & Pl. 5, Fig. 18 & Globorotalia plesiotumida \\
\hline Fig. 6.18 & Globanomalina pseudomenardii & Pl. 3, Fig. 2 & Planorotalites pseudomenardii \\
\hline Fig. 6.19 & Globanomalina pseudomenardii & Pl. 3, Fig. 1 & Planorotalites pseudomenardii \\
\hline Fig. 6.20 & Morozovella velascoensis & Pl. 5, Fig. 1 & Morozovella velascoensis \\
\hline Fig. 6.21 & Morozovella velascoensis & Pl. 5, Fig. 2 & Morozovella velascoensis \\
\hline Fig. 6.22 & Morozovella formosa formosa & Pl. 5, Fig. 9 & Morozovella subbotinae \\
\hline Fig. 6.23 & Morozovella formosa formosa & Pl. 5, Fig. 8 & Morozovella subbotinae \\
\hline Fig. 6.24 & Subbotina pseudobulloides & Pl. 4, Fig. 6 & Subbotina pseudobulloides \\
\hline
\end{tabular}




\begin{tabular}{|l|l|l|l|}
\hline \multicolumn{2}{|l|}{ Imam (2001) } & \multicolumn{2}{l|}{ Petters (1983) } \\
\hline Fig. 6.25 & Subbotina triloculinoides & Pl. 4, Fig. 4 & Subbotina triloculinoides \\
\hline Fig. 6.26 & Subbotina velascoensis & Pl. 4, Fig. 1 & Subbotina velascoensis \\
\hline
\end{tabular}

\section{Conclusions}

The author of this fraud pretended he was illustrating material he supposedly collected in remote areas of both Egypt and Libya (which makes quality control of the data difficult). However most photomicrographs were "borrowed" from the existing publications of other authors. The microfossils illustrated were all found to be characteristic of other stratigraphic intervals than those they were originally associated with in the plagiarised papers. In addition, M.M. Imam used classical (coralline red algae) or reference (charophytes) illustrations, but rotated (charophytes) or mirrored (planktonic foraminifers) some images, thus demonstrating that he has no real experience/competence in either of these fields of micropaleontology. Though the denunciation of the fraud was given some publicity ([Aguirre, 2004], [Bosch, 2004a], [Bosch, 2004b] and [Granier et al., 2007]), the falsified data have already begun to pollute science (for instance (Jackson et al., 2005) and (Jackson et al., 2006), who used the Imam publications to ascribe a time range to a tectonic event). Finally, as in the Gupta fraud ([Talent et al., 1988] and [Talent, 1989]), the most regrettable aspect is that it tarnishes the reputation of countless honest and professionally ethical colleagues from the same African-Middle-Eastern region.

\section{References}

Aguirre, 2004 J. Aguirre, Plagiarism in palaeontology. A new threat within the scientific community, Revista Española de Micropaleontología 36 (2) (2004), pp. 349-352.

Bosch, 2004a Bosch, X., 2004a. Fallout from fraud. The Scientist 5 (1), 20040922-02 $<$ http://www.the-scientist.com/news/20040922/02/>.

Bosch, 2004b Bosch, X., 2004b. Plagiarism in paleontology. The Scientist, Philadelphia 5 (1), 20041008-03 <http://www.the-scientist.com/news/20041008/03/>.

Feist and Ringeade, 1977 M. Feist and M. Ringeade, Étude biostratigraphique et paléobotanique (Charophytes) des formations continentales d'Aquitaine, de l'Éocène supérieur au Miocène inférieur, Bulletin de la Société géologique de France XIX (2) (1977), pp. 341-354.

Feist-Castel, 1977 M. Feist-Castel, Étude floristique et biostratigraphique des Charophytes dans les séries du Paléogène de Provence, Géologie méditerranéenne IV (2) (1977), pp. 109-138. 
Grambast and Grambast-Fessard, 1981 L. Grambast and N. Grambast-Fessard, Étude sur les Charophytes tertiaires d'Europe Occidentale. III - Le genre Gyrogona, Paléobiologie Continentale XII (2) (1981), pp. 1-35.

Grambast-Fessard, 1980 N. Grambast-Fessard, Les Charophytes du Montien de Mons (Belgique), Review of Palaeobotany and Palynology 30 (1980), pp. 67-88.

Granier et al., 2007 Granier, B., Feist, M., Bucur, I.I., Senowbari-Daryan, B., 2007. Further investigation of the, Imam's micropaleontological fraud: The stolen algae. In: Grgasović, T., Vlahović, I. (Eds.), Field Trip Guidebook and Abstracts - 9th International Symposium on Fossil Algae, Croatia, pp. 223-224.

Imam, 1999 M.M. Imam, Lithostratigraphy and planktonic foraminiferal biostratigraphy of the Late Eocene-Middle Miocene sequence in the area between Wadi Al Zeitun and Wadi Al Rahib, Al Bardia area, northeast Libya, Journal of African Earth Sciences 28 (3) (1999), pp. 619-639.

Imam, 2001 M.M. Imam, Biostratigraphy of the Upper Cretaceous-Lower Eocene succession in the Bani Walid area, northwest Libya, Journal of African Earth Sciences 33 (1) (2001), pp. 69-89.

Jackson et al., 2005 C.A.L. Jackson, R.L. Gawthorpe, I.D. Carr and I.R. Sharp, Normal faulting as a control on the stratigraphic development of shallow marine syn-rift sequences, Sedimentology 52 (2) (2005), pp. 313-338.

Jackson et al., 2006 C.A.L. Jackson, R.L. Gawthorpe, C.W. Leppard and I.R. Sharp, Riftinitiation development of normal fault blocks: insights from the Hammam Faraun fault block, Journal of the Geological Society 163 (2006), pp. 165-183.

Petters, 1983 S.W. Petters, Gulf of Guinea planktonic foraminiferal biochronology and geological history of the South Atlantic, Journal of foraminiferal Research 13 (1) (1983), pp. $32-59$.

Phillip et al., 1997 G. Phillip, M.M. Imam and G.I. Abdel Gawad, Planktonic foraminiferal biostratigraphy of the Miocene sequence in the area between Wadi ElTayiba and Wadi Sidri, west central Sinai, Egypt, Journal of African Earth Sciences 25 (3) (1997), pp. 435-451.

Refaat and Imam, 1999 A.A. Refaat and M.M. Imam, The Tayiba Red Beds: transitional marine-continental deposits in the precursor Suez Rift, Sinai, Egypt, Journal of African Earth Sciences 28 (3) (1999), pp. 487-506.

Talent, 1989 J.A. Talent, The case of the peripatetic fossils, Nature 338 (1989), pp. 613615. 
Talent et al., 1988 J.A. Talent, R.K. Goel, A.K. Jain and J.W. Pickett, Silurian and Devonian of India, Nepal and Bhutan: biostratigraphic and palaeobiogeographic anomalies, Courier Forschungsinstitut Senckenberg 106 (1988), pp. 1-57.

Waters and Snyder, 1986 V.J. Waters and S.W. Snyder, Planktonic foraminiferal biostratigraphy of the Pungo River Formation, southern Onslow Bay, North Carolina continental shelf, Journal of foraminiferal Research 16 (1) (1986), pp. 9-23.

Youssef et al., 1988 E.A.A. Youssef, S.E. Fahmy and M. Imam, Stratigraphy and microfacies of the Miocene sequence at Gebel Sarbut El Gamal, west-central Sinai, Egypt, Neues Jahrbuch für Geologie und Paläontologie, Abhandlungen 177 (1988), pp. 225-242. 